\title{
Estrategias familiares frente a la crisis de covid-19 en la ciudad de Santo Domingo - Ecuador: un estudio descriptivo
}

\author{
Fernanda Pilaguano Almachi[1], Carlos Quijano Mendoza[1], Carlos Troya \\ Altamirano[2] \\ 1. Clínica Nuestra Señora de Guadalupe, ASECU, Ecuador \\ 2. Universidad de Las Américas, ASECUP, Ecuador \\ 3. Ministerio de Salud Pública, ASECUP, Ecuador \\ 4. Pontificia Universidad Católica del Ecuador, SuportaMed, ASECUP, Ecuador
}

Doi: https://doi.org/10.23936/pfr.v5i3.167

PRÁCTICA FAMILIAR RURAL | Vol.5 | No.3 | Noviembre 2020 | Recibido: 10/09/2020 | Aprobado: 26/11/2020

\begin{abstract}
Cómo citar este artículo
Pilaguano, F., Quijano, C., Troya, C. Estrategias familiares frente a la crisis de covid-19 en la ciudad de Santo Domingo - Ecuador: un estudio descriptivo. Práctica Familiar. 2020 noviembre; 5(3).
\end{abstract}

Compartir en:

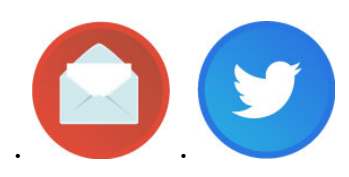

\section{Resumen}

Objetivo: Identificar las estrategias que las familias usan para enfrentar la crisis de la pandemia del covid-19 cuando tienen al menos un integrante con una enfermedad crónico degenerativa.

Metodología: Estudio descriptivo, transversal, cuantitativo, dirigido a un universo infinito, con una muestra aleatoria. Se aplicó una encuesta en formato digital donde se recogieron datos sociodemográficos, estrategias familiares y se midió la el afrontamiento mediante la versión modificada de la escala francesa "Echèlle Toulousaine de Coping". Los datos fueron procesados en el paquete estadístico SPSS versión 20.0. Se aplicaron medidas de estadística descriptiva, el test de chi cuadrado y el índice de correlación de Spearman.

Resultados: Hubo 134 participantes. La edad promedio fue de 30 años. La mayoría fueron mujeres $(\mathrm{n}=86(64.2 \%))$. La mayoría de las familias no realizaron cambios orientados a la protección de las personas con enfermedades crónicas (asilamiento, cambio de domicilio). La respuesta individual de afrontamiento fue pasiva $(\mathrm{n}=90,67.2 \%)$. La estrategia más usada para la evaluación de salud fue "el uso de alternativas que 
evitaron el contacto con los profesionales" $(n=67,50 \%)$. Menos del $10 \%$ de los participantes reportó que se haya abandonado el tratamiento $(\mathrm{n}=6,4.5 \%) ; \mathrm{y}$ cerca de la quinta parte $(\mathrm{n}=29,21.6 \%)$ usó tratamientos de medicina alternativa para las enfermedades descritas. El tipo de afrontamiento (activo o pasivo) fue independiente del sexo, la edad, y el tipo de vivienda. Un afrontamiento pasivo estuvo relacionado con aquellos participantes que no estaban desempleados. No hubo correlación significativa entre la edad y el nivel de afrontamiento $(\mathrm{Ro}=$ $0.058)$.

Conclusiones: Las estrategias individuales y familiares predominantes fueron de tipo pasivo. La disponibilidad de un empleo influyó en el afrontamiento pasivo. No existe relación directa entre el afrontamiento personal y las estrategias familiares.

Palabras clave: afrontamiento, estrategias familiares, covid-19

\title{
Family strategies in the face of the covid- 19 crisis in the city of Santo Domingo - Ecuador: a descriptive study
}

\begin{abstract}
Objective: To identify the strategies that families use to face the crisis of the covid-19 pandemic when they have at least one member with a chronic degenerative disease.

Methodology: Descriptive, cross-sectional, quantitative study, directed to an infinite universe, with a random sample. A survey was applied in digital format where sociodemographic data, family strategies were collected and coping was measured using the modified version of the French "Echèlle Toulousaine de Coping" scale. The data were processed in the statistical package SPSS version 20.0. Descriptive statistics measures, the chi square test and the Spearman correlation index were applied.

Results: There were 134 participants. The average age was 30 years. The majority were women $(n=86(64.2 \%))$. Most of the families did not make changes aimed at protecting people with chronic diseases (isolation, change of address). The individual coping response was passive $(\mathrm{n}=90,67.2 \%)$. The most used strategy for health evaluation was "the use of alternatives that avoided contact with professionals" $(\mathrm{n}=$ $67,50 \%)$. Less than $10 \%$ of the participants reported that treatment had been abandoned $(n=6,4.5 \%)$; and about a fifth $(n=29,21.6 \%)$ used alternative medicine treatments for the diseases described. The type of coping (active or passive) was independent of sex, age, and type of home. Passive coping was related to those participants who were not unemployed. There was no significant correlation between age and level of coping $(\mathrm{Ro}=0.058)$.

Conclusions: The predominant individual and family strategies were passive. The availability of a job influenced passive coping. There is no direct relationship between personal coping and family strategies.
\end{abstract}

Keywords: coping, family strategies, covid-19

\section{Introducción}

La familia es un conjunto de personas que comparten vínculos afectivos, consanguinidad o adopción, en un mismo espacio con normas, reglas y valores socioculturales en los que se desenvuelven.(1-6) La salud familiar es la salud y bienestar de los integrantes de la familia en relación a la dinámica interna, cumplimiento de funciones y adaptación al cambio; que ayudan al crecimiento y 
desarrollo en cada etapa de la vida, que puede afectar y estar afectado por su entorno, por ello deben mantenerse en equilibrio para obtener un buen estado de salud.(2,6-8)

Las dimensiones de la salud familiar son: el clima social familiar, que hace referencia a la relación interna con cada uno de sus miembros; la integridad familiar que está en relación al grado unión los miembros de la familia; el funcionamiento familiar que guía la satisfacción de las necesidades de los miembros en las etapas del ciclo vital para lograr el desarrollo; la resistencia familiar corresponde a los mecanismos de defensa frente a adversidades de los miembros; y, el afrontamiento familiar que es la capacidad de enfrentar acontecimientos o sucesos estresantes en la familia. $(2,9-11)$

La salud familiar se ve alterada por cualquier situación que afecte la salud individual de sus miembros, de igual forma al encontrase en una vida familiar no saludable va a enfermar a los miembros de la familia. Por lo tanto, el afrontamiento familiar es la manera de encontrar recursos, implementar acciones, que ayuden a superar acontecimientos estresantes. $(7,8,11)$

Las estrategias de afrontamiento se centran en las fuerzas cognitivas y comportamentales para protegerse y evitar cambios negativos, así como también cambiar el momento estresante y de dificultad. $(5,7,8)$ Las estrategias que la familia encuentre para enfrentar situaciones problemáticas van a estar en relación con el tiempo de aparición, el tipo de causante, su severidad, la perturbación que ocasione en la dinámica familiar, es decir las estrategias de afrontamientos van hacer dinámicas y se van modificando a lo largo del tiempo. $(7,8,12,13)$

La manera de afrontamiento de un individuo va a depender de los recursos que disponga, las creencias, las destrezas sociales, el apoyo social y los recursos materiales. $(5,14)$ Los estudios acerca del afrontamiento individual y familiar destacan los hallazgos de una relación positiva del afrontamiento con la salud y la funcionalidad del sistema familiar. $(5,14,15)$

Durante la pandemia por Covid-19 y como parte del confinamiento se emitieron una serie de recomendaciones de medidas no farmacológicas, basados en la experiencia de China y Corea del Sur. Dichas medidas tuvieron la finalidad de evitar la propagación de la Covid-19, e incluyeron el aislamiento social, restricción y cierre de fronteras, cierre de escuelas y universidades, entre los países que se adscribieron a estas medidas estuvo Ecuador. Una medida de especial interés fue el aislamiento de los adultos mayores o de las personas denominadas con vulnerabilidad, es decir aquellos con enfermedades crónico degenerativas. (16)

En vista de lo descrito el objetivo de este estudio fue identificar las estrategias que las familias de la ciudad de Santo Domingo usaron para enfrentar la crisis de la pandemia del covid-19 cuando tuvieron al menos un integrante con una enfermedad crónico degenerativa (consideradas como parte de las enfermedades que conferían vulnerabilidad para la forma grave de la enfermedad).

\section{Metodología}

Se trata de un estudio descriptivo, cuantitativo, dirigido a un universo infinito, con una muestra aleatoria. Se contactó a los posibles participantes, se los invitó a participar luego de explicar de qué se trataba el estudio. Se desarrolló una encuesta orientada a identificar la respuesta familiar frente a la crisis sanitaria y también hacia la respuesta individual del participante. Los datos fueron procesados en el paquete estadístico SPSS versión 20.0. Se aplicaron medidas de estadística descriptiva y el test de chi cuadrado.

La encuesta se aplicó en formato digital autollenado, se aplicó en el periodo entre abril y septiembre de 2020. Estuvo constituida por tres bloques de preguntas, el primer grupo de preguntas estuvo dirigido a recoger datos sociodemográficos de los participantes (edad, sexo, ocupación, nivel de instrucción, etc.). El siguiente grupo 
de preguntas indagó sobre la presencia de personas con enfermedades crónico degenerativas en la familia y sobre estrategias que se habían recomendado para evitar el contagio de Covid-19. El último bloque de preguntas fue relacionado con la capacidad de afrontamiento a nivel individual.

Para identificar las estrategias familiares se consultó si la familia adoptó alguna de las estrategias recomendadas por los organismos internacionales para evitar el contagio de las personas con enfermedades crónico degenerativas como son el aislamiento de la persona enferma, cambio de domicilio de la persona enferma o ninguno. Lo propio se hizo con relación a la conducta de la familia frente al manejo de la enfermedad del familiar con un problema de salud.

La capacidad de afrontamiento (individual) se midió mediante la aplicación de una versión modificada de la escala francesa "Echèlle Toulousaine de Coping". $(6,8) \mathrm{La}$ Escala modificada permite identificar dos factores: Afrontamiento Pasivo (reactivos $1,2,3,4,6,7,12,16,18,21,24,26$ ) y Afrontamiento Activo (reactivos 5, 8, 9, 10 , $11,13,14,15,17,19,20,22,23,25)$.

El afrontamiento activo se refiere a comportamientos de acción directa sobre el problema, búsqueda de información, estrategias de anticipación a un desastre, control de sí mismo y de las circunstancias, y de soporte social. El afrontamiento pasivo se refiere, en contraste, a comportamientos de rechazo y negación del evento, retraimiento y aceptación pasiva y se mide en escala Likert de 1 al 5. (8)La escala mide las capacidades de afrontamiento individuales, la interacción entre la persona y el medio ambiente pero no incluye la capacidad de acción comunitaria. (8)

\section{Resultados}

Un total de 134 participantes completaron el cuestionario. La edad promedio de los respondedores fue de 30 años. La mayoría de los participantes fueron mujeres $(n=86$ $(64.2 \%))$, y el nivel de instrucción predominante fue de instrucción superior $(n=92$, $68.7 \%$ ), la mayoría describieron su estado de ocupación como desempleado ( $\mathrm{n}=72$, $53.7 \%)$, y el tipo de vivienda predominante fue propia $(n=80,59.7 \%)$. Un detalle mayor del análisis de la población estudiada está disponible en la Tabla 1.

La presencia de al menos una enfermedad crónica degenerativa se dio en el siguiente orden: hipertensión arterial $(\mathrm{n}=54)$, diabetes $(\mathrm{n}=35)$, cardiopatía $(\mathrm{n}=24)$, enfermedad pulmonar $(\mathrm{n}=15)$, enfermedad renal $(\mathrm{n}=13)$, algún tipo de cáncer $(\mathrm{n}=$ 2). La mayoría de los participantes respondieron que sus familias no realizaron cambios orientados a la protección de las personas con enfermedades crónicas ni al inicio ni durante la pandemia (asilamiento, cambio de domicilio). Solo 17 de los participantes señalaron no contar con una persona de su círculo familiar que cuente con una persona con una enfermedad de las descritas.

En el análisis de la respuesta individual la mayoría de los participantes describieron su conducta dentro del afrontamiento pasivo $(n=90,67.2 \%)$. En el componente familiar la mayoría de los participantes señaló que "la familia no modificó nada" tanto al inicio como en el transcurso de la pandemia $(n=101,75.4 \%$ y $n=99$, $73.9 \%$ ) a pesar de que había al menos un integrante de la familia con una enfermedad crónico degenerativa. Al consultar sobre la manera "como se hacía la evaluación sobre el estado de salud del integrante enfermo" la estrategia más usada fue "el uso de alternativas que evitaron el contacto con los profesionales" ( $n=67$, $50 \%$ ). Menos del $10 \%$ de los participantes reportó que se haya abandonado el tratamiento $(n=6,4.5 \%)$; y cerca de la quinta parte $(n=29,21.6 \%)$ usó tratamientos de medicina alternativa para las enfermedades descritas. Las estrategias de afrontamiento se describen mejor en la tabla 2.

El análisis de fiabilidad de la versión modificada de la escala francesa "Echèlle Toulousaine de Coping" arrojó un alfa de Cronbach de 0.842. El análisis factorial de la escala fue sometido a la prueba de KMO que arrojó un valor de 0.809 , de igual 
manera se midió el índice de Bartlett con un valor de 0.000. Dicho análisis arrojó dos factores como se pude apreciar en la tabla 3 .

Con relación al análisis bivariado se estableció si había independencia entre el puntaje de afrontamiento y algunas variables sociodemográficas como: tipo de vivienda, número de personas en la vivienda, la presencia de alguna de las enfermedades crónico degenerativas descritas. También se comparó los dos niveles de afrontamiento, es decir el afrontamiento pasivo o activo de los participantes comparado con el uso de algunas estrategias familiares (i.e., aislamiento de la persona enferma, cambio de domicilio de la persona enferma).

\section{Tabla 1. Análisis univariado}

Variable

Frecuencia

Porcentaje

Sexo

Masculino

48

35.8

Femenino

86

64.2

Instrucción educativa

Sin instrucción

0.7

Primaria

Secundaria

26.9

Superior

36

68.7

Ocupación

Desempleado

72

53.7

Comerciante o cuentapropista

6

4.5

Empleado público

14

10.4

Empleado privado

42

31.3

Tipo de vivienda

Arrendada

39

29.1

Prestada

10

7.5

Propia

80

59.7

Otra

5

3.7

Edad de participantes

Menores de 20 años

21-40 años

41-60 años

21

Más de 60 años

2

15.7

1.5

Presencia de integrantes con enfermedades

Hipertensión

Cardiopatías

Enfermedad pulmonar

Enfermedades renales 

Variable
Frecuencia
Porcentaje

Afrontamiento (nivel individual)

Afrontamiento pasivo

Medidas al inicio de la pandemia

Aislamiento en un cuarto de la (s) persona 27

(s) con enfermedades como las descritas antes

Cambio de domicilio de la (s) persona (s) con alguna de las enfermedades descritas antes

La familia no modificó nada en un primer momento

Medidas durante la pandemia

Aislamiento en un cuarto de la (s) persona 30

(s) con enfermedades como las descritas antes

Cambio de domicilio de la (s) persona (s) con alguna de las enfermedades descritas antes

La familia no modificó nada en un primer momento

Evaluación de salud del integrante enfermo

Se acudió a consulta con el médico regular 20

Se evitó ir al médico en absoluto (no salió 37

de casa)

Se hizo atender con otro médico

Se usó alternativas que evitaron el contacto con los profesionales (teléfono, wassapp, internet)

El tipo de afrontamiento (activo o pasivo) fue independiente del sexo, la edad, y el tipo de vivienda (Tabla 4) medido con la prueba de chi cuadrado. En cambio, un tipo de afrontamiento pasivo estuvo relacionado con aquellos participantes que no estaban desempleados (Tabla 4). El tipo de afrontamiento pasivo (individual) estuvo más relacionado con estrategias similares (no hacer nada) empleadas por las familias de estos participantes (Tabla 4). De igual manera para el análisis bivariado se analizó la correlación entre la edad de los participantes y el afrontamiento, pero no hubo correlación significativa medida con el cociente de correlación de Spearman $(\mathrm{Ro}=0.058)$. 
25. Trato de no precipitarme y de reflexionar sobre los pasos a $\quad 0,766 \quad-0,107$ seguir

17. Me fijo objetivos y redoblo esfuerzos

$0,752 \quad 0,012$

20. Reflexiono sobre las estrategias a utilizar

$0,743 \quad-0,040$

14. Hago modificaciones en mi entorno para evitar un desastre

$0,729 \quad-0,078$

9. Consulto sobre el problema con profesionales

$0,720 \quad 0,016$

8. Busco información con personas que saben

$0,710 \quad-0,017$

15. He establecido mi propio plan de prevención y lo pongo en

0,704

$-0,009$ marcha

13. Hago frente directamente a la situación

$0,701 \quad-0,049$

23. Trato de cambiar mis hábitos de vida en función del problema

$0,700 \quad 0,119$

22. Tengo un plan preventivo y lo sigo

$0,646 \quad-0,264$

10. Controlo en todo momento mis emociones

$0,611 \quad 0,023$

5. Analizo las circunstancias para saber qué hacer

$0,591-0,011$

19. Participo más en actividades de prevención civil

$0,523 \quad 0,104$

11. Hablo con mi familia para compartir emociones

$0,510 \quad 0,049$

7. Busco actividades para pensar en otra cosa

$0,506 \quad 0,328$ 


\section{Tabla 4. Análisis bivariado}

$\begin{array}{lccccc} & \begin{array}{c}\text { Afrontamiento } \\ \text { activo }\end{array} & (\%) & \begin{array}{c}\text { Afrontamiento } \\ \text { pasivo }\end{array} & (\%) & \text { P valor } \\ & & & & & \\ \text { Sexo masculino } & 14 & (29.2) & 34 & (70.8) & 0.499 \\ \text { Sexo femenino } & 30 & (34.9) & 56 & (65.1) & \\ & & & & & \\ & 14 & (40) & 21 & (60) & 0.511 \\ \text { Hasta 20 años } & 24 & (31.6) & 52 & (68.4) & \\ \text { Más de 40 años } & 6 & (26.1) & 17 & (73.9) & \\ & & & & & \\ & & & & & \\ \text { Desempleado } & 30 & (41.7) & 42 & (58.3) & 0.015^{*} \\ \text { No desempleado } & 14 & (22.6) & 48 & (77.4) & \end{array}$


Vivienda propia

Otro tipo de vivienda
(35)

(29.6)
52

38
(65)

(70.4)

\section{Discusión}

En un estudio realizado en realizado Andalucía, España, se evaluó la intensidad del duelo y estrategias de afrontamiento posterior a la pérdida de su empleo, se concluyó que los participantes con mayores dificultades económicas experimentaron un duelo con mayor intensidad e incrementaron el riesgo de padecer algún deterioro de la salud durante la etapa de desempleo. Es así que las personas que experimentan un duelo más intenso utilizan con mayor frecuencia medidas pasivas basadas en negación, autoinculpación, autodestrucción y destrucción, siendo propio de la misma condición la perdida de la esperanza lo que pospone búsqueda de soluciones activas en un periodo de crisis, estos resultados son similares al presente estudio, donde las medidas pasivas prevalecen en la mayoría de los casos. Sin embargo, en el presente trabajo no se exploraron las razones para la implementación de dichas estrategias. (17)

Un estudio de Aguilar-Mendoza hecho en la zona metropolitana de San Salvador, el Salvador, evaluó el afrontamiento asociado antes del surgimiento de eventos de desastres naturales. No hubo diferencias estadísticas significativas en el afrontamiento activo entre hombres y mujeres, ni tampoco se las identificó con relación al estado civil. Tampoco se encontraron diferencias en el afrontamiento que presentan los participantes según la edad. Y el mayor el porcentaje de familias presentaron un afrontamiento pasivo. El presente estudio tiene similares resultados con relación al sexo y la edad. (8)

En otro estudio, hecho la Habana Cuba, en familias y en pacientes con diagnóstico clínico de epilepsia, el afrontamiento familiar a la epilepsia se manifestó en comportamientos que fueron desde la sobreprotección del miembro enfermos hasta el reforzamiento de su supuesta discapacidad, siendo más frecuentes las respuestas activas a diferencia de nuestro estudio que resalta en varias condiciones las respuestas pasivas por partes de las familias(18)

\section{Conclusiones}

Las estrategias individuales y familiares en los hogares donde había al menos una persona enferma, durante la pandemia por Covid-19 fueron de tipo pasivo. La respuesta activa o pasiva, estaría relacionada con el grado de incertidumbre que experimenta la familia pues si se trata de un escenario en el que la familia tiene experiencia (manejo de una paciente con una enfermedad) predomina el afrontamiento activo; en cambio, en situación contrarias (desempleo, la posibilidad de un desastre, la pandemia por Covid-19), es decir con alta incertidumbre predominan el afrontamiento pasivo.

La disponibilidad de un empleo estuvo relacionada con la conducta de pasividad si se compara con el desempleo, lo cual es consecuente con la disponibilidad de recursos para enfrentar la crisis. Esto cuestiona la idoneidad de las medidas de control no farmacológico que han sido difundidas ampliamente durante la duración de la crisis sanitaria, bajo la premisa "Quédate en casa". Pues serían medidas idóneas para un sector de la población (el que tiene empleo y por lo tanto recursos para enfrentar las dificultades).

No existe relación directa entre el afrontamiento personal y las estrategias familiares, pues como se aprecia en el presente estudio, existe ambigüedad en las respuestas, mientras en el nivel individual se reconoce un afrontamiento pasivo, pero en el nivel familiar se responde (cambio de estrategia para mantener atención médica). Se requiere profundizar mayor caracterización de las características de las familias participantes para poder extraer mejores conclusiones de trabajos similares. 


\section{Referencias bibliográficas}

1. Armas N, Díaz Muñoz L. Entre Voces y silencios, Las Familias por dentro, Guía para el trabajo con familias. 2017; Available from: http://familiats.com.mx/wpcontent/uploads/2017/06/Armas2007.pdf

2. Rodriguez JSL, Serrano ML, Picon NJ, Sanchez ID. Consistencia Interna Y Validez De Un Cuestionario Para Medir La Autopercepcion Del Estado De Salud Familiar. Rev Esp Salud Publica. 2012;86(5):509-21.

3. García Méndez M, Rivera Aragón S, Reyes Lagunes I, Díaz Loving R. Construcción de una escala de funcionamiento familiar Construction of a family functioning scale. Ridep. 2006;2(22):91-110.

4. Pujol AV. Repercusiones Psicológicas en la Familia ante la Enfermedad Grave y Crónica del Niño. :1-12.

5. Jiménez M, Amarís M, Valle M. Afrontamiento en crisis familiares : El caso del divorcio cuando se tienen hijos adolescentes Coping in family crisis : Divorce with teenager' s children. Salud Uninorte. 2012;28:99-112.

6. López Vázquez E, Marván ML. Validación de una escala de afrontamiento frente a riesgos extremos. Salud Publica Mex. 2004;46(3):216-21.

7. Martínez-Montilla JM, Amador-Marín B, Guerra-Martín MD. Estrategias de afrontamiento familiar y repercusiones en la salud familiar: Una revisión de la literatura. Enfermería Glob. 2017;16(3):576.

8. Aguilar Mendoza AS. El afrontamiento y la capacidad de resiliencia de las personas ante los riesgos por desastres naturales. Entorno. 2016;(62):34-46.

9. Jiménez Tallón M, Ferro M, Gómez Gómez R, Parra López P. Evaluación del clima familiar en una muestra de adolescentes. Vol. 52, Revista de psicología general y aplicada: Revista de la Federación Española de Asociaciones de Psicología. 1999. p. 453-62.

10. Aguayo Martínez A, López Vásquez E, Lorenzo Ruiz A, Ávila Guerrero ME, Vera Jiménez JA, Hindrichs I. E $\mathrm{s} t \mathrm{r}$ a $\mathrm{t}$ e $\mathrm{g}$ i a $\mathrm{s} d \mathrm{e}$ a f $\mathrm{r}$ o $\mathrm{n} \mathrm{t}$ a $\mathrm{m}$ i e $\mathrm{n} \mathrm{t}$ o a $\mathrm{n} \mathrm{t}$ e emergencias y desastres. Cuad Cris y emergencias [Internet]. 2004;15(2). Available from:

http://cuadernosdecrisis.com/docs/2016/numero15vol2_2016_4Estretegias_afrontamientos_desastres.pc 11. Molina Pérez MJ, Rodríguez Chinchilla N. Estrategias de afrontamiento: un programa de entrenamiento para paramédicos de la Cruz Roja. Rev Costarric Psicol. 2011;30(45):17-33.

12. Uribe Meneses A. Características familiares en situación de enfermedad crónica features family living in chronic disease. 2014;19(1).

13. Quintero F, Amaris M del C, Pacheco RA. Afrontamiento y funcionamiento en familias en situación de discapacidad Coping and functioning in families with a disability situation. 2020;(1987).

14. Arteaga A C. Pobreza y Estrategias Familiares: Debates y Reflexiones. Rev Mad. 2007;(C):144-64.

15. Oliveira O, Ariza M. Pobreza Estudios Demográficos y Urbanos. 2007;22.

16. Patiño Lugo DF, Vélez M, Velásquez Salazar P, Vera Giraldo CY, Vélez V, Marín

$\mathrm{IC}$, et al. Intervenciones no farmacológicas para la contención, mitigación y supresión de la infección por COVID-19. Sintésis rápida TT - Non-pharmacological interventions for containment, mitigation and suppression of COVID-19 infection. Rapid Synthesis. Colomb Med [Internet]. 2020;35. Available from: http://fiadmin.bvsalud.org/document/view/p8yzj

17. Díaz C, Navarro Abal Y, Climent JA, Ortega E, López López MJ, Casado J. Pérdida y Afrontamiento en Desempleados: Adaptación del Inventario Texas Revisado de Duelo a la Situación de Pérdida de Empleo. Rev Iberoam Diagnostico y Eval Psicol. 2016;2(42):111-24.

18. Fabelo Roche JR, Iglesias Moré S, Louro Bernal I, Gonzáles Pal S. Afrontamiento familiar a la epilepsia Family coping with epilepsy. Rev Cuba Salud Pública. 2013;3939(33):450-61. 